\title{
2-Oxoglutarate: linking TCA cycle function with amino acid, glucosinolate, flavonoid, alkaloid, and gibberellin biosynthesis
}

\author{
Wagner L. Araújo ${ }^{1,2}$, Auxiliadora O. Martins ${ }^{1}$, Alisdair R. Fernie ${ }^{3 *}$ and Takayuki Tohge ${ }^{3}$ \\ ${ }^{1}$ Departamento de Biologia Vegetal, Universidade Federal de Viçosa, Viçosa, Brazil \\ ${ }^{2}$ Max-Planck Partner Group at the Departamento de Biologia Vegetal, Universidade Federal de Viçosa, Viçosa, Brazil \\ ${ }^{3}$ Max-Planck-Institut für Molekular Pflanzenphysiologie, Potsdam-Golm, Germany
}

Edited by:

Stefan Martens, Edmund Mach

Foundation, Italy

Reviewed by:

Soren Bak, University of Copenhagen, Denmark

Fumiya Kurosaki, University of

Toyama, Japan

\section{*Correspondence:}

Alisdair R. Fernie, Max-Planck-Institut für Molekular Pflanzenphysiologie, Am Mühlenberg 1, 14476 Potsdam-Golm, Brandenburg, Germany

e-mail: fernie@mpimp-golm.mpg.de
The tricarboxylic acid (TCA) cycle intermediate 2-oxoglutarate (2-OG) is used as an obligatory substrate in a range of oxidative reactions catalyzed by 2-OG-dependent dioxygenases. These enzymes are widespread in nature being involved in several important biochemical processes. We have recently demonstrated that tomato plants in which the TCA cycle enzyme 2-OG dehydrogenase (2-ODD) was antisense inhibited were characterized by early senescence and modified fruit ripening associated with differences in the levels of bioactive gibberellin (GA). Accordingly, there is now compelling evidence that the TCA cycle plays an important role in modulating the rate of flux from 2-OG to amino acid metabolism. Here we discuss recent advances in the biochemistry and molecular biology of 2-OG metabolism occurring in different biological systems indicating the importance of 2-OG and 2-OG dependent dioxygenases not only in glucosinolate, flavonoid and alkaloid metabolism but also in GA and amino acid metabolism. We additionally summarize recent findings regarding the impact of modification of 2-OG metabolism on biosynthetic pathways involving 2-ODDs.

Keywords: dioxygenases, 2-oxoglutarate dependent dioxygenases, gibberellin, TCA cycle, flavonoid, alkaloid

\section{INTRODUCTION}

2-Oxoglutarate (2-OG), a key organic acid of the tricarboxylic acid (TCA) cycle (Lancien et al., 2000; Scheible et al., 2000), is also an obligatory substrate for 2-OG-dependent dioxygenases (2-ODDs), as depicted in Reaction 1. Briefly, dioxygenases can be defined as enzymes catalyzing reactions in which both atoms of molecular oxygen are incorporated into substrates (Figure 1). In the hydroxylation reaction catalyzed by dioxygenases, one atom of molecular oxygen is incorporated into the substrate, while the other atom of oxygen is incorporated into 2-OG resulting in the subsequent formation of succinate and the release of carbon dioxide.

The 2-ODDs are considered the largest known family of nonheme oxidizing enzymes (Prescott and John, 1996; Ozer and Bruick, 2007; Kawai etal., 2014). Members of this family are found throughout biology catalyzing a number of oxidation reactions and have been identified in many organisms ranging from prokaryotes to eukaryotes. Furthermore, oxidative reactions catalyzed by 2-ODD are involved in biosynthetic processes leading to materials of medicinal or agrochemical importance including collagen or other modified polypeptides and amino acids, plant secondary metabolites, phytohormones such as ethylene and gibberellins (GAs) as well as $\beta$-lactam antibiotics, i.e., penicillins and cephalosporins (Vaillancourt et al., 2006; Loenarz and Schofield, 2008; Martens et al., 2010 and references therein). Altogether this indicates that 2-ODD and its substrate, 2-OG, are highly important in plant metabolism as a whole and thus a massive impact of their genetic modification on plant metabolism in different plant tissues is expected. It is worth mentioning that a difficulty impeding metabolic engineering within this enzyme family is the fact that multiple pathways within the metabolic network could be affected, linking the enzymes to the specific target metabolites.

Here we discuss recent advances in the biochemistry and molecular biology of 2-OG metabolism occurring in different biological systems indicating the importance of 2-OG and 2-ODDs not only in glucosinolate, flavonoid, and alkaloid metabolism but also in GA and amino acid metabolism.

\section{THE IMPORTANCE OF 2-OXOGLUTARATE METABOLISM IN HIGHER PLANTS}

2-Oxoglutarate participates in a range of reactions in distinct plant cell compartments (Weber and Flügge, 2002; Foyer et al., 2003), also being a key metabolite at the crossroads of carbon/nitrogen metabolism as it is required for ammonia assimilation (Hodges, 2002). Despite this fact, it still remains rather unclear where the major site of production of 2-OG resides. This organic acid can be produced from either sugar respiration or amino acid transamination following the concerted action of isocitrate dehydrogenases, aminotransaminases, and glutamate dehydrogenases (Lancien et al., 2000). We have previously demonstrated that the mitochondrial enzyme 2-OG dehydrogenase (OGDH) has an important role in 2-OG production and metabolism, controlling the levels of this important organic acid in plant cells (Araújo et al., 2008, 2012a,b). These results in conjunction with others in which similar reduction in the TCA cycle activity was also demonstrated (Sienkiewicz-Porzucek et al., 2008, 2010) indicates 


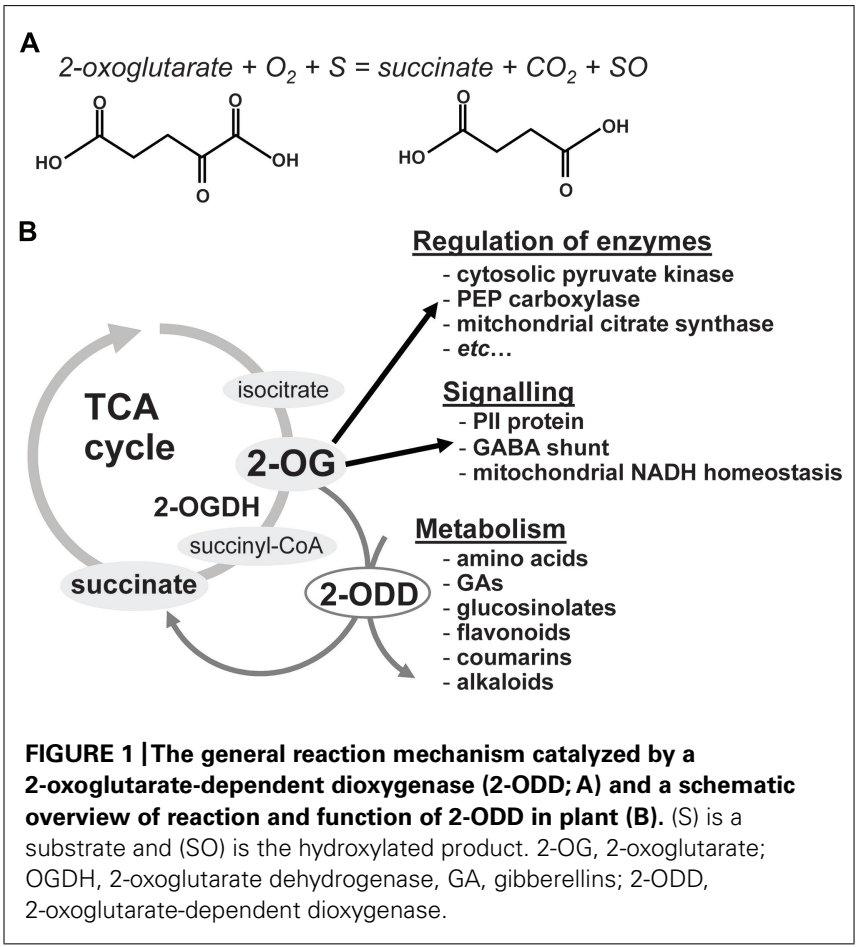

that mitochondrial TCA cycle enzymes contribute considerably to the regulation of nitrogen assimilation in leaves and that a substantial portion of 2-OG production occurs in the mitochondria itself. It is worth mentioning that the exact balance between the use of cytosolic versus mitochondrial routes for synthesis of 2-OG remains rather unknown. Thus the discovery that the carbon for nitrogen assimilation in leaves comes from carbon stored from the previous night (most likely organic acids such as citrate stored in the vacuole and released to the cytosol; Gauthier et al., 2010) raises the possibility that 2-OG for nitrogen assimilation is generated predominantly in the cytosol and therefore mitochondrial enzymes make only a quantitatively minor contribution. It is important to note that 2-OG is also a direct regulator of several enzymes (e.g., cytosolic pyruvate kinase and PEP carboxylase, mitochondrial citrate synthase, and alternative oxidase) associated with sugar and/or organic acid flux and redox control between cytosol and mitochondria (for a review see Hodges, 2002).

In addition to this, 2-OG has itself been suggested to play a role as a signal metabolite in plants (Lancien et al., 2000; Ferrario-Mery etal., 2001; Feria Bourrellier et al., 2009). This role is, however, largely based on analogy to the important role it plays in conjuncture with the plastidial PII protein in plants (Uhrig et al., 2009). Reports to date suggest that whilst PII may regulates a small number of enzyme systems in plants including $N$-acetyl-glutamate kinase (Ferrario-Mery et al., 2006; Feria Bourrellier etal., 2009) and plastidial acetyl-CoA carboxylase (Feria Bourrellier et al., 2010) its role is unlikely to be as pivotal in plants as in non-plant systems(Araujo et al., 2012). That said it is clear that the production of 2-OG in the mitochondria or cytosol is an important determinant of some plastidial activities.

\section{ON THE CONNECTIONS BETWEEN 2-OXOGLUTARATE METABOLISM AND GIBBERELLIN IN HIGHER PLANTS}

Much effort has been expended on elucidating the physiological functions of the various genes regulated by GA (Yamaguchi, 2008). However, studies concerning the associated effects of GA on energy metabolism and growth are rare. This fact notwithstanding, characterization of the pyruvate dehydrogenase kinase 1 (PDK1) has demonstrated that GA modulates the activity of the mitochondrial pyruvate dehydrogenase by regulating PDK1 expression and controlling growth in rice (Yazaki et al., 2003; Jan et al., 2006). Collectively it also indicates that GA might modify primary metabolism at the entry point of TCA cycle. In addition it has been demonstrated by the overexpression of genes associated with GA biosynthesis or catabolism that GA levels play key roles on transcriptional programs influencing plant growth (Biemelt et al., 2004; Dayan et al., 2010). Furthermore, reduction of TCA cycle enzymatic activity has led to reduction of GA levels in tomato roots (van der Merwe et al., 2009).

In this vein tomato plants with reduced levels of the TCA cycle enzyme 2-OGDH were recently characterized by early leaf senescence and a modified fruit ripening most likely due to differences in the levels of bioactive GAs (Araújo et al., 2012b). Given that the reduction in the activity of this enzyme was associated to a higher impact on respiration rates than observed previously in other TCA enzyme it seems reasonable to suggest that 2-OG might be of critical importance in the regulation of respiration rates in higher plants. It should be mentioned that both the chemical (Araújo etal., 2008) and molecular (Araújo et al., 2012b) inhibition of 2-OGDH was characterized by significant alterations in both sugars and TCA cycle intermediates. Notably, a compensatory augmentation in the flux of the GABA shunt was clearly observed most likely in an attempt to restore the TCA cycle. In addition, the changes in the GABA shunt suggest that its up regulation is needed to maintain succinate supply to the mitochondrial electron transport chain. This finding highlights the metabolic importance of the GABA shunt in plants (Fait et al., 2008) and is similar to findings observed following neuronal OGDH inhibition (Sá Santos et al., 2006; Shi et al., 2009). Remarkably this compensatory up regulation of the GABA shunt was coupled with significant shifts in cellular pools of both nitrate and amino acids in general. Specifically, metabolite profiling of the OGDH antisense lines demonstrated that steady state levels of photorespiratory intermediates, namely glycerate, and glycine, were reduced, coupled with a significant reduction in the label redistribution to glycine and serine (Araújo et al., $2012 \mathrm{~b})$. These results suggest that the down-regulation of the TCA cycle activity was integrated with an up-regulation in the flux through the photorespiration pathway as part of a reprogramming to maintain either mitochondrial NADH homeostasis and/or the glutamate pool size. NADH and NADPH levels play an important role in mitochondrial respiratory metabolism and it can explain the maintenance of the levels of both NAD and $\mathrm{NADPH}$ in OGDH antisense plants (Araújo et al., 2012b). Altogether the results of this work coupled with others described above have clearly demonstrated that the alteration of the mitochondrial 2-OG metabolism has greater impacts in plant respiration and its connections than previously expected. It is important 
to mention that the precise nature of this interaction between 2-OG metabolism and hormone-mediated control of growth and senescence by GA remains an exciting topic for future research. For the purposes of this review perhaps most important is to outline the key role of 2-OG and by extension 2-ODD in GA metabolism.

It also presents metabolic evidence for a key role of 2-OG and 2-ODD in both GA metabolism. The synthetic enzymes involved in GAs biosynthesis have been well-characterized (Graebe, 1987). In GA biosynthesis, several types of oxidation enzymes are involved in GA biosynthesis, such as ent-kaurene oxidase (AtKO, CYP701A1) and ent-kaurenoic acid oxidase (AtKAO, CYP88A3, and CYP88A4) types, GA- $\beta$-hydroxylase and GA20-oxidase (2ODD) types (Yamaguchi, 2008; Kawai et al., 2014). By great efforts of former works in GA metabolism, several 2-ODDs involved in GA biosynthesis have been found in several plant species (Prescott, 1993; Figure 2A); for example, $2 \beta$-hydoxylase(s) from Phaseolus vulgaris (Griggs et al., 1991) and from Pisum sativum (Smith and Macmillan, 1986), 2 $\beta$-hydoxylase(s) from P. vulgaris (Smith et al., 1990), GA20-hydroxylase from P. sativum (Lange and Graebe, 1989) and from Cucurbita maxima (Lange and Graebe, 1989). Half of the reactions in GA biosynthesis are mainly converted by 2-ODDs (Hedden etal., 1982; Prescott, 1993). The identification and characterization of the 2-ODDs involved in
A

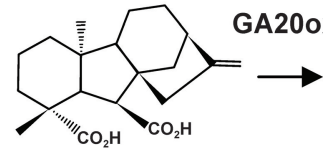

$\mathrm{GA}_{12}$

B<smiles>CS(=O)CCCCC([O-])=[N+]([O-])[O-]</smiles>
methylsulfinylalkyl glucosinolate

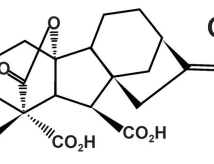

$\mathrm{GA}_{9}$

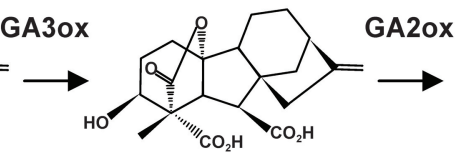

$\mathrm{GA}_{4}$

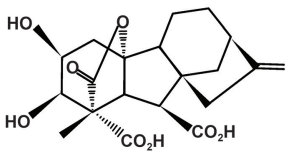

$\mathrm{GA}_{34}$<smiles>O=C(O)CCCCC(=O)O[Na]</smiles>

alkenyl glucosinolate<smiles>C=CC(O)CC(=NC(=O)O)[N+](=O)[O-]</smiles>

hydroxyalkenyl glucosinolate

AOP3

hydroxyalkyl glucosinolate

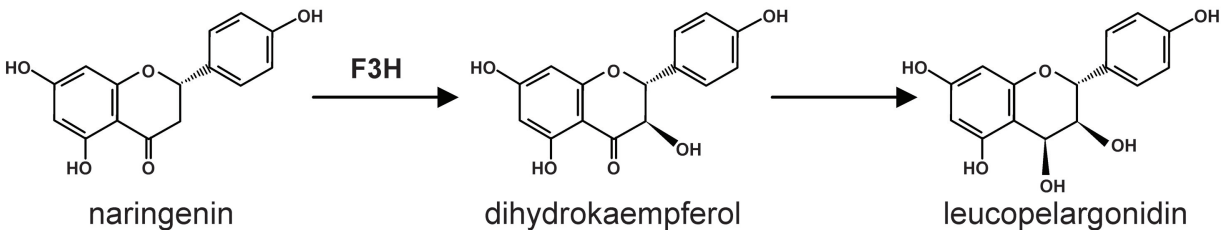<smiles>CC[C@@H](Oc1ccc(O)cc1)Oc1cc(O)cc(O)c1</smiles>
dihydrokaempferol leucopelargonidin

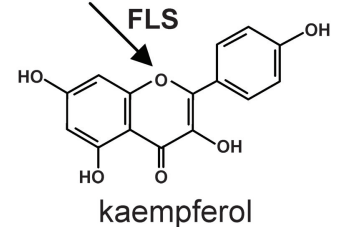

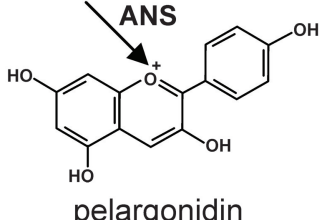

D<smiles>CN1C2C=CC1CC(OC(=O)[C@H](CO)C1=CCCCC1)C2</smiles><smiles>CN1CCC(OC(=O)[C@H](CO)c2ccccc2)CC1O</smiles><smiles>CN1C2CCCC1CC(OC(=O)[C@@H](CO)c1ccccc1)C2</smiles>

$E$

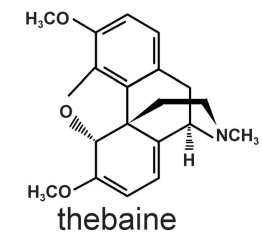<smiles>COc1ccc2c3c1O[C@H]1C(O)=CC=C4[C@H](C2)N(C)CC[C@]431</smiles>

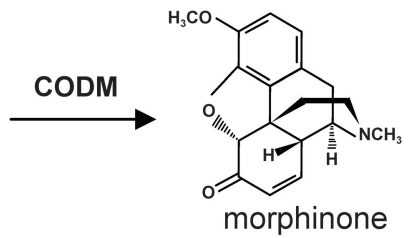

FIGURE 2 | Major enzymatic reactions of 2-ODD involved in GA and plant secondary metabolism. 2-ODDs involved in (A) GA, (B) glucosinolate, (C) flavonoid, (D) tropan alkaloid, (E) isoquinorine biosynthesis are described. GA20ox, GA20-oxidase; GA3ox, GA33-hydroxylase; GA2ox, GA 2-oxidase; AOP, alkenyl/hydroxy $(\mathrm{OH})$ alkyl producing enzymes; GSL-OH, glucosinolate 2-oxoacid-dependant dioxygenase gene; FS-I, flavone synthase I; FLS, flavonol synthase, $\mathrm{F} 3 \mathrm{H}$, flavanone 3-hydroxylase, ANS, anthocyanin synthase; $\mathrm{H} 6 \mathrm{H}$, hyoscyamine 6-hydroxylase; T6ODM, 6-demethylase; CODM, codeine O-demehtylase. 
GA biosynthesis has also led to major advances in the elucidation of the pathway including understanding of chemical diversity of GAs. Furthermore, it seems reasonable to consider that a part of GA biosynthesis is controlled by 2-OG content in plant.

\section{THE METABOLIC IMPORTANCE OF 2-ODD IN THE REGULATION OF SECONDARY METABOLISM}

The oxygenase which leads to the incorporation of oxygen atoms from molecular oxygen is one of the most important enzymes in terms of conferring variation within plant secondary metabolism. Two functionally different classes of oxygenases, namely cytochrome P450 enzymes and 2-ODD, are involved in several pathways of plant secondary metabolism namely hydroxycinnamates, flavonoids and alkaloids (Tohge et al., 2013). As for GA biosynthesis, almost half of the oxidation reactions which are involved in late steps of their biosynthesis are catalyzed by 2-ODD (Tohge et al., 2013). In the biosynthesis of glucosinolates which are nitrogen/sulfur-containing secondary metabolites mostly found in Brassica species, three 2-ODDs, namely alkenyl /hydroxy $(\mathrm{OH})$ alkyl producing enzymes (AOP1, AOP2, AOP3; Kliebenstein et al., 2001) and glucosinolate 2-oxoacid-dependent dioxygenase gene (GSL-OH; Hansen et al., 2008) have been found in Arabidopsis (Figure 2B). Since GSL-OH is evolutionarily highly distant from AOP2, it has been suggested that GSL-OH represents an independent recruitment from a different 2-ODD clade to the same metabolism (Kawai et al., 2014).

In flavonoid biosynthesis, four types of 2-ODDs; flavonol synthase (FLS), flavanone 3-hydroxylase (F3H), anthocyanin synthase (ANS also known as LDOX), and flavone synthase I (FS-I) are characterized as key enzymes in late steps of flavonoid aglycone formations resulting to in species specific flavonoid profiles (Figure 2C; Martens et al., 2001; Turnbull et al., 2004; Tohge et al., 2013). Flavonols are catalyzed from flavanone by hydroxylation of carbon-3 and 2,3-dehydration by F3H and FLS, respectively. On the other hand, ANS which catalyses the formation of dihydroflavonols from leucoanthocyanidins is known to be one of as one of the key enzymes involved in red pigmentation via anthocyanin and proanthocyanidin biosynthesis. FS-I which is found mainly in monocot and Apiaceae species uses flavanone as the substrate for flavone backbone formation. Given that F3H provides the precursor of substrates for FLS and ANS, it has been suggested that the evolution of FLS and ANS occurred after the emergence of $\mathrm{F} 3 \mathrm{H}$ during 2-ODD evolution in seed plants (Stafford, 1991). Phylogenetic tree analysis using gene family of FLS, F3H, and ANS from several plant species revealed clear separation between FLS, F3H, and ANS as well as monocots, dicots and leguminous species as subclade of FLS, F3H, and ANS. This fact may suggest that FLS, F3H, and ANS have evolved in early stages of evolution (Tohge et al., 2013). In the other branches of phenolic secondary metabolism such as coumarin and 2,4-di-hydroxy-2H-1,4-benzoxazin-3(4H)one (DIBOA) biosynthesis, several 2-ODDs have been found as key enzymes in their biosynthesis namely, p-coumaroylCoA 2'-hydroxylase ( $\mathrm{C}^{\prime} \mathrm{H}$; Vialart et al., 2012), feruloyl-CoA 6'-hydroxylase (F6'H; Kai et al., 2008), and DIBOA-7-hydroxylase (BX6; Frey et al., 2003).
Several 2-ODDs are also involved in the synthesis of different alkaloids, which is the second largest class of plant secondary metabolism, have been characterized in several plant species. In the biosynthesis of tropane alkaloid, hyoscyamine 6-hydroxylase $(\mathrm{H} 6 \mathrm{H})$ in scoporamine biosynthesis of Hyoscyamus niger (Figure 2D; Matsuda et al., 1991), Anisodus tanguticus (Liu etal., 2005), and Atropa belladonna (Suzuki et al., 1999), $2^{\prime}$-deoxymugineic-acid 2'-dioxygenase (IDS3) in mugineic acid biosynthesis of Hordeum vulgare (Nakanishi et al., 2000) have been characterized. In addition, two 2-ODDs involved the biosynthesis of morphine namely thebaine 6-demethylase (T6ODM) and codeine O-demethylase (CODM) have been found form Papaver somniferum (Figure 2E; Hagel and Facchini, 2010).

\section{SUMMARY}

In summary, 2-OG is not only a TCA cycle intermediate but also a co-factor for a diverse range of enzymes involved in amino acid, glucosinolate, flavonoid, alkaloid, and GA metabolism. Specifically in the case of GA and amino acid, recent evidence indicates that 2-OG levels control the rate of their biosynthesis. Further work is, however, needed to establish it also for glucosinolate, flavonoid, and alkaloid biosynthesis. Moreover, whilst preliminary studies have been attempted to understand evolutionary origins of the 2-ODD, more comprehensive analysis of this will be required to deepen our understanding of this important gene family.

\section{ACKNOWLEDGMENTS}

This work was supported by funding from the Max Planck Society (to Wagner L. Araújo, Takayuki Tohge, and Alisdair R. Fernie) and the National Council for Scientific and Technological Development (CNPq-Brazil, Grant 483525/2012-0 to Wagner L. Araújo). Research fellowships granted by $\mathrm{CNPq}$ to Wagner L. Araújo are also gratefully acknowledged.

\section{REFERENCES}

Araújo, W. L., Nunes-Nesi, A., Nikoloski, Z., Sweetlove, L. J., and Fernie, A. R. (2012a). Metabolic control and regulation of the tricarboxylic acid cycle in photosynthetic and heterotrophic plant tissues. Plant Cell Environ. 35, 1-21. doi: 10.1111/j.1365-3040.2011.02332.x

Araújo, W. L., Tohge, T., Osorio, S., Lohse, M., Balbo, I., Krahnert, I., et al. (2012b). Antisense inhibition of the 2-oxoglutarate dehydrogenase complex in tomato demonstrates its importance for plant respiration and during leaf senescence and fruit maturation. Plant Cell 24, 2328-2351. doi: 10.1105/tpc.112. 099002

Araújo, W. L., Nunes-Nesi, A., Trenkamp, S., Bunik, V. I., and Fernie, A. R. (2008). Inhibition of 2-oxoglutarate dehydrogenase in potato tuber suggests the enzyme is limiting for respiration and confirms its importance in nitrogen assimilation. Plant Physiol. 148, 1782-1796. doi: 10.1104/pp.108.126219

Araujo, W. L., Tohge, T., Nunes-Nesi, A., Daloso, D. M., Nimick, M., Krahnert, I., et al. (2012). Phosphonate analogs of 2-oxoglutarate perturb metabolism and gene expression in illuminated Arabidopsis leaves. Front. Plant Sci. 3:114. doi: 10.3389/fpls.2012.00114

Biemelt, S., Tschiersch, H., and Sonnewald, U. (2004). Impact of altered gibberellin metabolism on biomass accumulation, lignin biosynthesis, and photosynthesis in transgenic tobacco plants. Plant Physiol. 135, 254-265. doi: 10.1104/pp.103.036988

Dayan, J., Schwarzkopf, M., Avni, A., and Aloni, R. (2010). Enhancing plant growth and fiber production by silencing GA 2-oxidase. Plant Biotechnol. J. 8, 425-435. doi: 10.1111/j.1467-7652.2009.00480.x

Fait, A., Fromm, H., Walter, D., Galili, G., and Fernie, A. R. (2008). Highway or byway: the metabolic role of the GABA shunt in plants. Trends Plant Sci. 13, 14-19. doi: 10.1016/j.tplants.2007.10.005 
Feria Bourrellier, A. B., Ferrario-Méry, S., Vidal, J., and Hodges, M. (2009). Metabolite regulation of the interaction between Arabidopsis thaliana PII and $\mathrm{N}$-acetyl-l-glutamate kinase. Biochem. Biophys. Res. Commun. 387, 700-704. doi: 10.1016/j.bbrc.2009.07.088

Feria Bourrellier, A. B., Valot, B., Guillot, A., Ambard-Bretteville, F., Vidal, J., and Hodges, M. (2010). Chloroplast acetyl-CoA carboxylase activity is 2-oxoglutarate-regulated by interaction of PII with the biotin carboxyl carrier subunit. Proc. Natl. Acad. Sci. U.S.A. 107, 502-507. doi: 10.1073/pnas. 0910097107

Ferrario-Mery, S., Besin, E., Pichon, O., Meyer, C., and Hodges, M. (2006). The regulatory PII protein controls arginine biosynthesis in Arabidopsis. FEBS Lett. 580, 2015-2020. doi: 10.1016/j.febslet.2006.02.075

Ferrario-Mery, S., Masclaux, C., Suzuki, A., Valadier, M. H., Hirel, B., and Foyer, C. H. (2001). Glutamine and alpha-ketoglutarate are metabolite signals involved in nitrate reductase gene transcription in untransformed and transformed tobacco plants deficient in ferredoxin-glutamine-alpha-ketoglutarate aminotransferase. Planta 213, 265-271. doi: 10.1007/s004250000504

Foyer, C. H., Parry, M., and Noctor, G. (2003). Markers and signals associated with nitrogen assimilation in higher plants. J. Exp. Bot. 54, 585-593. doi: 10.1093/jxb/erg053

Frey, M., Huber, K., Park, W. J., Sicker, D., Lindberg, P., Meeley, R. B., et al. (2003). A 2-oxoglutarate-dependent dioxygenase is integrated in DIMBOA-biosynthesis. Phytochemistry 62, 371-376. doi: 10.1016/S0031-9422(02)00556-3

Gauthier, P. P. G., Bligny, R., Gout, E., Mahé, A., Nogués, S., Hodges, M., et al. (2010). In folio isotopic tracing demonstrates that nitrogen assimilation into glutamate is mostly independent from current $\mathrm{CO} 2$ assimilation in illuminated leaves of Brassica napus. New Phytol. 185, 988-999. doi: 10.1111/j.1469-8137.2009. 03130.x

Graebe, J. E. (1987). Gibberellin biosynthesis and control. Annu. Rev. Plant Physiol. Plant Mol. Biol. 38, 419-465. doi: 10.1146/annurev.pp.38.060187.002223

Griggs, D. L., Hedden, P., and Lazarus, C. M. (1991). Partial-purification of 2 gibberellin 2-beta-hydroxylases from cotyledons of Phaseolus vulgaris. Phytochemistry 30, 2507-2512. doi: 10.1016/0031-9422(91)85090-M

Hagel, J. M., and Facchini, P. J. (2010). Dioxygenases catalyze the O-demethylation steps of morphine biosynthesis in opium poppy. Nat. Chem. Biol. 6, 273-275. doi: 10.1038/nchembio.317

Hansen, B. G., Kerwin, R. E., Ober, J. A., Lambrix, V. M., Mitchell-Olds, T., Gershenzon, J., et al. (2008). A novel 2-oxoacid-dependent dioxygenase involved in the formation of the goiterogenic 2-hydroxybut-3-enyl glucosinolate and generalist insect resistance in Arabidopsis. Plant Physiol. 148, 2096-2108. doi: 10.1104/pp.108.129981

Hedden, P., Phinney, B. O., Heupel, R., Fujii, D., Cohen, H., Gaskin, P., et al. (1982). Hormones of young tassels of Zea mays. Phytochemistry 21, 391-393. doi: 10.1016/S0031-9422(00)95273-7

Hodges, M. (2002). Enzyme redundancy and the importance of 2-oxoglutarate in plant ammonium assimilation. J. Exp. Bot. 53, 905-916. doi 10.1093/jexbot/53.370.905

Jan, A., Nakamura, H., Handa, H., Ichikawa, H., Matsumoto, H., and Komatsu, S, (2006). Gibberellin regulates mitochondrial pyruvate dehydrogenase activity in rice. Plant Cell Physiol. 47, 244-253. doi: 10.1093/pcp/pci241

Kai, K., Mizutani, M., Kawamura, N., Yamamoto, R., Tamai, M., Yamaguchi, H., et al. (2008). Scopoletin is biosynthesized via ortho-hydroxylation of feruloyl $\mathrm{CoA}$ by a 2-oxoglutarate-dependent dioxygenase in Arabidopsis thaliana. Plant $\mathrm{J}$. 55, 989-999. doi: 10.1111/j.1365-313X.2008.03568.x

Kawai, Y., Ono, E., and Mizutani, M. (2014). Evolution and diversity of the 2oxoglutarate-dependent dioxygenase superfamily in plants. Plant J. 78, 328-343. doi: $10.1111 /$ tpj.12479

Kliebenstein, D. J., Lambrix, V. M., Reichelt, M., Gershenzon, J., and Mitchell-Olds, T. (2001). Gene duplication in the diversification of secondary metabolism: tandem 2-oxoglutarate-dependent dioxygenases control glucosinolate biosynthesis in Arabidopsis. Plant Cell 13, 681-693. doi: 10.1105/tpc. 13.3.681

Lancien, M., Gadal, P., and Hodges, M. (2000). Enzyme redundancy and the importance of 2-oxoglutarate in higher plant ammonium assimilation. Plant Physiol. 123, 817-824. doi: 10.1104/pp.123.3.817

Lange, T., and Graebe, J. E. (1989). The partial purification and characterization of a gibberellin C-20 hydroxylase from immature Pisum sativum L. seeds. Planta 179, 211-221. doi: 10.1007/BF00393691
Liu, T., Zhu, P., Cheng, K. D., Meng, C., and Zhu, H. X. (2005). Molecular cloning and expression of putrescine $\mathrm{N}$-methyltransferase from the hairy roots of Anisodus tanguticus. Planta Med. 71, 987-989. doi: 10.1055/s-2005-871260

Loenarz, C., and Schofield, C. J. (2008). Expanding chemical biology of 2-oxoglutarate oxygenases. Nat. Chem. Biol. 4, 152-156. doi: 10.1038/nchembio0308-152

Martens, S., Forkmann, G., Matern, U., and Lukacin, R. (2001). Cloning of parsley flavone synthase I. Phytochemistry 58, 43-46. doi: 10.1016/S0031-9422(01) 00191-1

Martens, S., Preuß, A., and Matern, U. (2010). Multifunctional flavonoid dioxygenases: flavonol and anthocyanin biosynthesis in Arabidopsis thaliana L. Phytochemistry 71, 1040-1049. doi: 10.1016/j.phytochem.2010.04.016

Matsuda, J., Okabe, S., Hashimoto, T., and Yamada, Y. (1991). Molecular cloning of hyoscyamine 6-beta-hydroxylase, a 2-oxoglutarate dependent dioxygenase from cultured roots of Hyoscyamus niger. J. Biol. Chem. 266, 9460-9464.

Nakanishi, H., Yamaguchi, H., Sasakuma, T., Nishizawa, N. K., and Mori, S. (2000). Two dioxygenase genes, Ids3 and Ids2, from Hordeum vulgare are involved in the biosynthesis of mugineic acid family phytosiderophores. Plant Mol. Biol. 44, 199-207. doi: 10.1023/A:1006491521586

Ozer, A., and Bruick, R. K. (2007). Non-heme dioxygenases: cellular sensors and regulators jelly rolled into one? Nat. Chem. Biol. 3, 144-153. doi: 10.1038/nchembio863

Prescott, A. G. (1993). A dilemma of dioxygenases (or where biochemistry and molecular biology fail to meet). J. Exp. Bot. 44, 849-861. doi: 10.1093/jxb/44.5.849 Prescott, A. G., and John, P. (1996). Dioxygenases: molecular structure and role in plant metabolism. Annu. Rev. Plant Physiol. Plant Mol. Biol. 47, 245-271. doi: 10.1146/annurev.arplant.47.1.245

Sá Santos, S., Gibson, G. E., Cooper, A. J. L., Denton, T. T., Thompson, C. M., Bunik, V. I., et al. (2006). Inhibitors of the $\alpha$-ketoglutarate dehydrogenase complex alter [1-13C] glucose and [U-13C]glutamate metabolism in cerebellar granule neurons. J. Neurosci. Res. 83, 450-458. doi: 10.1002/jnr.20749

Scheible, W. R., Krapp, A., and Stitt, M. (2000). Reciprocal diurnal changes of phosphoenolpyruvate carboxylase expression and cytosolic pyruvate kinase, citrate synthase and NADP-isocitrate dehydrogenase expression regulate organic acid metabolism during nitrate assimilation in tobacco leaves. Plant Cell Environ. 23, 1155-1167. doi: 10.1046/j.1365-3040.2000. 00634.x

Shi, Q., Risa, Ø., Sonnewald, U., and Gibson, G. E. (2009). Mild reduction in the activity of the alpha-ketoglutarate dehydrogenase complex elevates GABA shunt and glycolysis. J. Neurochem. 109, 214-221. doi: 10.1111/j.14714159.2009.05955.x

Sienkiewicz-Porzucek, A., Nunes-Nesi, A., Sulpice, R., Lisec, J., Centeno, D. C., Carillo, P., et al. (2008). Mild reductions in mitochondrial citrate synthase activity result in a compromised nitrate assimilation and reduced leaf pigmentation but have no effect on photosynthetic performance or growth. Plant Physiol. 147, 115-127. doi: 10.1104/pp.108.117978

Sienkiewicz-Porzucek, A., Sulpice, R., Osorio, S., Krahnert, I., Leisse, A., Urbanczyk-Wochniak, E., et al. (2010). Mild reductions in mitochondrial NADdependent isocitrate dehydrogenase activity result in altered nitrate assimilation and pigmentation but do not impact growth. Mol. Plant 3, 156-173. doi: 10.1093/mp/ssp101

Smith, V. A., Gaskin, P., and Macmillan, J. (1990). Partial purification and characterization of the gibberellin A20 3-beta-hydroxylase from seeds of Phaseolus vulgaris. Plant Physiol. 94, 1390-1401. doi: 10.1104/pp.94.3.1390

Smith, V. A., and Macmillan, J. (1986). The partial purification and characterisation of gibberellin 2 beta -hydroxylases from seeds of Pisum sativum. Planta 167, 9-18. doi: 10.1007/BF00446362

Stafford, H. A. (1991). Flavonoid evolution - an enzymatic approach. Plant Physiol. 96, 680-685. doi: 10.1104/pp.96.3.680

Suzuki, K., Yun, D. J., Chen, X. Y., Yamada, Y., and Hashimoto, T. (1999). An Atropa belladonna hyoscyamine 6 beta-hydroxylase gene is differentially expressed in the root pericycle and anthers. Plant Mol. Biol. 40, 141-152. doi: 10.1023/A:1026465518112

Tohge, T., Watanabe, M., Hoefgen, R., and Fernie, A. R. (2013). The evolution of phenylpropanoid metabolism in the green lineage. Crit. Rev. Biochem. Mol. Biol. 48, 123-152. doi: 10.3109/10409238.2012.758083

Turnbull, J. J., Nakajima, J.-I., Welford, R. W. D., Yamazaki, M., Saito, K., and Schofield, C. J. (2004). Mechanistic studies on three 2-oxoglutarate-dependent 
oxygenases of flavonoid biosynthesis: anthocyanidin synthase, flavonol synthase, and flavanone 3 $\beta$-hydroxylase. J. Biol. Chem. 279, 1206-1216. doi: 10.1074/jbc.M309228200

Uhrig, R. G., Ng, K. K. S., and Moorhead, G. B. G. (2009). PII in higher plants: a modern role for an ancient protein. Trends Plant Sci. 14, 505-511. doi: 10.1016/j.tplants.2009.07.003

Vaillancourt, F. H., Bolin, J. T., and Eltis, L. D. (2006). The ins and outs of ring-cleaving dioxygenases. Crit. Rev. Biochem. Mol. Biol. 41, 241-267. doi: $10.1080 / 10409230600817422$

van der Merwe, M. J., Osorio, S., Moritz, T., Nunes-Nesi, A., and Fernie, A. R. (2009). Decreased mitochondrial activities of malate dehydrogenase and fumarase in tomato lead to altered root growth and architecture via diverse mechanisms. Plant Physiol. 149, 653-669. doi: 10.1104/pp.108.130518

Vialart, G., Hehn, A., Olry, A., Ito, K., Krieger, C., Larbat, R., et al. (2012). A 2-oxoglutarate-dependent dioxygenase from Ruta graveolens L. exhibits pcoumaroyl CoA $2^{\prime}$-hydroxylase activity $\left(\mathrm{C}^{\prime} \mathrm{H}\right)$ : a missing step in the synthesis of umbelliferone in plants. Plant J. 70, 460-470. doi: 10.1080/10409230600817422

Weber, A., and Flügge, U. I. (2002). Interaction of cytosolic and plastidic nitrogen metabolism in plants. J. Exp. Bot. 53, 865-874. doi: 10.1093/jexbot/53.370.865

Yamaguchi, S. (2008). Gibberellin metabolism and its regulation. Annu. Rev. Plant Biol. 59, 225-251. doi: 10.1146/annurev.arplant.59.032607.092804
Yazaki, J., Kishimoto, N., Nagata, Y., Ishikawa, M., Fujii, F., Hashimoto, A., et al. (2003). Genomics approach to abscisic acid- and gibberellin-responsive genes in rice. DNA Res. 10, 249-261. doi: 10.1093/dnares/10.6.249

Conflict of Interest Statement: The authors declare that the research was conducted in the absence of any commercial or financial relationships that could be construed as a potential conflict of interest.

Received: 12 August 2014; accepted: 26 September 2014; published online: 15 October 2014.

Citation: Araújo WL, Martins AO, Fernie AR and Tohge T (2014) 2-Oxoglutarate: linking TCA cycle function with amino acid, glucosinolate, flavonoid, alkaloid, and gibberellin biosynthesis. Front. Plant Sci. 5:552. doi: 10.3389/fpls.2014.00552

This article was submitted to Plant Metabolism and Chemodiversity, a section of the journal Frontiers in Plant Science.

Copyright () 2014 Araújo, Martins, Fernie and Tohge. This is an open-access article distributed under the terms of the Creative Commons Attribution License (CC BY). The use, distribution or reproduction in other forums is permitted, provided the original author(s) or licensor are credited and that the original publication in this journal is cited, in accordance with accepted academic practice. No use, distribution or reproduction is permitted which does not comply with these terms. 